\title{
Comparison of Characteristics and Clinical Outcomes in 27-Gauge versus 23-Gauge Vitrectomy Surgery
}

This article was published in the following Dove Press journal: Clinical Ophthalmology

\author{
Omar A Saleh (D) \\ Saif A Alshamarti (iD ${ }^{2}$ \\ Nakhleh E Abu-Yaghi $\mathbb{D D}^{3}$ \\ 'Department of Ophthalmology, Jordan \\ University of Science and Technology, \\ Irbid, Jordan; ${ }^{2}$ Department of \\ Ophthalmology, College of Medicine, \\ University of Al-Qadisiyah, Diwanyah, \\ Iraq; ${ }^{3}$ Department of Special Surgery/ \\ Ophthalmology Division, School of \\ Medicine, The University of Jordan, \\ Amman, Jordan
}

Purpose: To compare characteristics, clinical outcomes and complication profiles of a cohort of patients undergoing 27-gauge versus 23-gauge vitrectomy surgery at a tertiary medical center in the Middle East.

Methods: This is a retrospective comparative study of indication-matched patients undergoing 27gauge versus 23-gauge pars plana vitrectomy for a set of predetermined diagnoses. Data including indications for surgery, clinical exams, visual outcomes, and complications were documented and analyzed.

Results: A total of 22 patients who had 27-gauge pars plana vitrectomy were compared with 44 patients who had 23-gauge surgery for matched indications by a single retina surgeon. Mean follow-up was 14 months after surgery. Indications included macular pucker, macular hole, and vitreous hemorrhage. Both the rate of hypotony at postoperative day 1 and the need for sclerotomy stitching at the end of surgery were significantly more frequent in 23-gauge compared to 27 -gauge cases $(25 \%$ in 23 -gauge compared to $0 \%$ in 27 -gauge $(\mathrm{p}=0.012)$ and $18 \%$ in the 23 -gauge compared to $0 \%$ in the 27 -gauge $(\mathrm{p}=0.045)$, respectively). The mean duration of surgery was similar among groups (35 mins in the 27-gauge group versus 39 mins in the 23-gauge group $(\mathrm{p}=0.116)$ ), and all eyes achieved anatomic success. In the 27 gauge group, best-corrected visual acuity improved from a mean of 20/90 at baseline to a mean of $20 / 40$ at 1 month ( $p=0.005$ ) and in the 23-gauge group from a mean of 20/95 to 20/ $50(\mathrm{p}=0.001)$ with no statistical difference between the groups.

Conclusion: In this cohort of patients undergoing vitrectomy at a tertiary eye center, the rate of hypotony and the need for sclerotomy suturing were significantly less in the 27-gauge group versus the 23-gauge group. Surgical outcomes were similar in both study groups. The use of the novel 27-gauge instrument system is useful in selected vitreoretinal diseases and appears comparable to the widely used 23 -gauge system.

Keywords: micro-incisional eye surgery, pars plana vitrectomy, sclerotomy, hypotony

\section{Introduction}

Trans-conjunctival micro-incisional vitrectomy surgery (MIVS) has gained a great momentum since the first introduction of 23- and 25-gauge instrument systems almost two decades ago. Such smaller gauge vitrectomy systems have advantages over the conventional 20-gauge system including faster wound healing, earlier visual recovery, decreased operative time, lower complication rates, decreased surgically-induced trauma, improved patient's comfort level and decreased postoperative inflammation. ${ }^{1-5}$
Correspondence: Omar A Saleh Department of Ophthalmology,Jordan University of Science and Technology, 3030 Ramtha Street, Irbid 221 I0, Jordan $\mathrm{Tel}+962-754-1594$

Email omarsaleh80@yahoo.com 
Some of these advantages may be attributed to the selfsealing sclerotomies in MIVS which should eliminate the need for conjunctival dissection and scleral and conjunctival suturing. Nonetheless, sutureless sclerotomies have been associated with post-operative hypotony and choroidal detachment in 23- and 25-gauge compared with 20gauge vitrectomy. 6 ,7

The recent introduction of 27 -gauge vitrectomy systems has been regarded as an important step towards more minimally invasive surgical practice. The application of this platform may be useful in various vitreoretinal conditions and may offer potentially more secure selfsealing sclerotomies.

In our report, we study 27-gauge and 23-gauge vitrectomy surgeries performed for several vitreoretinal indications. We compare the two platforms in terms of the need for sclerotomy stitches and the rate of post-operative hypotony, clinical outcomes, duration of surgery and complication profiles.

\section{Methods}

\section{Study Design and Data Collection}

We were given permission by the institutional review board at the University of Al-Qadisiyah, Diwaniyah, Iraq to conduct this retrospective comparative review of patients who had 27-gauge and 23-gauge vitrectomy at a university-affiliated ambulatory eye surgery center between March 2016 and June 2018. All patients provided written informed consent. We identified 22 consecutive patients who had 27-gauge vitrectomy and compared them to 44 consecutive and indication-matched 23-gauge vitrectomy cases within the same time period. For all participants, existing medical records were reviewed, and no patients' identifiers were kept in record.

Data collected included demographic information, diagnosis/indication for surgery, Snellen best-corrected visual acuity (BCVA), intraocular pressure (IOP) measured by Goldmann tonometry and lens status. Additional information documented included gauge of vitrectomy surgery, intra-operative and post-operative complications, surgical instruments, total operative time (from the insertion of the lid speculum at the start to its removal at the end) and duration of follow-up after surgery. The last visit before surgery was considered the baseline visit, and the last visit in the follow-up of the patient was considered the final visit. The early postoperative period was defined as the first week postoperatively. Hypotony was defined as IOP of $5 \mathrm{mmHg}$ or less.
For purposes of statistical analysis, Snellen BCVA measurements were converted to LogMAR units.

Exclusion criteria included follow-up of less than 1 year after surgery, incomplete or missing medical records, cases who had phacoemulsification with the vitrectomy surgery and cases who had prior vitrectomy. Primary study outcomes were the need for sclerotomy stitches at the end of surgery and the rate of hypotony in the early postoperative period. The need for sclerotomy stitching was determined at the conclusion of surgery after the surgeon attempted to adequately inflate the eye with air. The presence of air bubbles leaking through the sclerotomy was checked and/or the failure to maintain an adequate pressure inside the eye was judged by the surgeon's index finger. Secondary outcomes included changes in BCVA and IOP and complications associated with surgery.

\section{Statistics}

Data were collected on an electronic database using Microsoft Excel 2013 (Microsoft, Redmond, WA). For statistical analysis, two-tailed Fisher's exact test was used for categorical variables and unpaired Student's $t$-test was used for continuous variables. A p-value of $<0.05$ was accepted as statistically significant.

\section{Surgical Setting and Technique}

All surgeries were performed by one vitreoretinal surgeon (O. Saleh) with the same surgical setting for each of the 27or 23-gauge platforms. We used 27-gauge Total Plus vitrectomy sets (Constellation ${ }^{\circledR}$ Vision System, Alcon Laboratories, Inc., Fort Worth, TX, USA) and vitrectomy probes were attached to a vented gas-forced infusion system (OERTLI OS3 ${ }^{\circledR}$, Oertli Instrumente AG, Switzerland). Vitrectomy surgery included the standard 3-pars plana ports ( 1 port for infusion and 2 ports for intraocular instrumentation). After retrobulbar anesthesia, the eye was prepared under sterile conditions including conjunctival disinfection with $5 \%$ povidone. Starting with the infusion port, three pars plana ports were created using valved 23gauge (Alcon Inc.) and 27-gauge (Synergetics Inc.) trocar cannulas. All 23-gauge trocars were inserted in a beveled 2-step manner with a first partial insertion at an oblique angle of $15^{\circ}$ to $20^{\circ}$ tangential to the scleral surface followed by entry into the vitreous cavity at an angle of $30^{\circ}$ to $40^{\circ}$. For the insertion of the 27-gauge trocars, a 1-step insertion technique at about $30^{\circ}$ was employed. 
Vitrectomy parameters were set for proportional dualmode vitrectomy with cutting rate up to $1500 \mathrm{cpm}$ and aspiration rate up to $600 \mathrm{mmHg}$. A panoramic view with Resight $700^{\circledR}$ (Carl Zeiss Meditec AG, Germany) was used in all surgeries. Intravitreal triamcinolone acetonide was used in some cases to ease the visualization of vitreoretinal adhesion points if deemed necessary by the surgeon. Core vitrectomy was carried out and posterior hyaloid separation, if not already present, was performed by aspiration and mechanical lifting of the peripapillary cortical vitreous using the vitrectomy probe. End-gripping intraocular forceps and stepped laser probe of the appropriate gauge were used to peel epiretinal membranes (ERM) and the internal limiting membrane (ILM) and for panretinal laser photocoagulation, respectively. When needed, other 27-gauge or 23-gauge instruments were used as indicated. At the end of the surgery, depressed examination of the peripheral retina and vitreous base was done under the microscope to check for peripheral retinal breaks. When needed, depressed peripheral vitreous shaving was performed. Next, partial or complete fluid-air exchange was performed using the vitrector or the softtip cannula. The surgery was concluded by removing the trocars. Sclerotomies were checked for air leak and vicryl sutures were placed if needed. Topical antibiotic ointment was then applied, and the eye was covered with a patch and a shield.

\section{Results}

After applying the inclusion and exclusion criteria, a total of 22 patients in the 27-gauge group and 44 patients in the 23 -gauge group were included in the study. The demographic features, duration of follow-up time, and indications for vitrectomy surgery among the groups are depicted in Table 1. At the end of the vitrectomy surgery, significantly more patients in the 23-gauge group needed stitching for at least one sclerotomy: 11 patients from the 23 -gauge group (25\%) compared to none of the patients in the 27-gauge group $(\mathrm{p}=0.012)$. Six eyes needed stitching of two sclerotomies, 3 eyes needed stitching of one sclerotomy, and 2 eyes needed stitching of all three sclerotomies. Despite having sutures, 2 of these eyes developed hypotony anyway the next day. One of them had 2 sclerotomies sutured and the other had all 3 sclerotomies sutured at the end of surgery. Hypotony at postoperative day 1 was significantly more frequent in the 23-gauge group, with 8 eyes (18\%) recording an IOP $\leq 5 \mathrm{mmHg}$ compared to none of the eyes in the 27 -gauge
Table I Demographic Characteristics and Indications for Surgery in Patients Who Had 23-Gauge and 27-Gauge Vitrectomy

\begin{tabular}{|l|l|l|l|}
\hline & 27-Gauge Group & 23-Gauge Group & P value \\
\hline Number of patients & 22 & 44 & \\
Age in years & $59.2 \pm 13.7$ & $58.7 \pm 13.5$ & 0.879 \\
Gender & 12 female, 10 male & 20 female, 24 male & 0.603 \\
Follow-up in months & $14.9 \pm 2.1$ & $17.8 \pm 5.1$ & 0.011 \\
Lens status & $82 \%$ & $84 \%$ & 1.0 \\
(\% pseudophakia) & & & \\
\hline Indication for vitrectomy & & & \\
-ERM & $11(50 \%)$ & $23(52 \%)$ & 1.0 \\
-MH & $4(18 \%)$ & $8(18 \%)$ & 1.0 \\
-VH & $4(18 \%)$ & $7(16 \%)$ & 1.0 \\
-VMT & $2(9 \%)$ & $5(11 \%)$ & 1.0 \\
-Vitreous opacity & $1(5 \%)$ & $1(3 \%)$ & 1.0 \\
\hline
\end{tabular}

Notes: ${ }^{2}$ Vitreous hemorrhage was due to proliferative diabetic retinopathy in 3 cases in the 27-gauge group and 5 cases in the 23-gauge group, due to Terson's syndrome in one case in each group, and due to I case of not previously diagnosed branch retinal vein occlusion in the 23 -gauge group. ${ }^{\text {}}$ The patient with this condition in the 27 -gauge group had visually significant asteroid hyalosis and the patient in the 23-gauge group had vitreous opacities due to inactive intermediate uveitis.

Abbreviations: ERM, epiretinal membrane; $\mathrm{MH}$, macular hole; $\mathrm{VH}$, vitreous hemorrhage; VMT, vitreomacular traction.

group ( $\mathrm{p}=0.045)$. Although 2 eyes with hypotony had choroidal effusions, those were self-limiting. No hypotony or choroidal effusion was recorded in any of the patients starting at 1 week postoperatively and up until the final visit. In the 27 -gauge group, IOP did not significantly change from baseline to the post-operative period and up to the final visit. In contrast, eyes who had 23-gauge surgery had a significant decrease in mean IOP from $14 \mathrm{~mm} \mathrm{Hg}$ at baseline to $12 \mathrm{~mm} \mathrm{Hg}$ the first day postoperatively but returned near the baseline value at 1 week postoperatively (Table 2).

Up until the final visit, no intraoperative or postoperative complications were documented in the 27-gauge group. One intraoperative complication, however, an iatrogenic retinal tear, was documented in a macular hole case in the 23-gauge cohort. The tear was detected peripherally at the end of surgery upon scleral depression at 11 o'clock and was not associated with subretinal fluid. It was managed by vitreous base shaving and laser retinopexy with no postoperative implications recorded up to the final visit.

Mean total operative time was not significantly different between the two groups at $34.8 \pm 11.4$ mins (range 20-60) in the 27-gauge group and $38.8 \pm 8.6$ mins (range 25-56) in the 23 -gauge group $(p=0.116)$. All study eyes achieved anatomic success with successful removal of ERM or ILM and clearance of the vitreous blood or opacities. Mean BCVA recorded at 1 month after surgery was significantly 
Table 2 Intraocular Pressure Changes Before and After 27Gauge and 23-Gauge Vitrectomy

\begin{tabular}{|l|l|l|l|l|l|}
\hline & Baseline & $\begin{array}{l}\text { Postop } \\
\text { Day I }\end{array}$ & $\begin{array}{l}\text { Postop } \\
\text { Week I }\end{array}$ & $\begin{array}{l}\text { Final } \\
\text { Visit }\end{array}$ & P value $^{\mathbf{a}}$ \\
\hline 27-gauge & $14.1 \pm 3.2$ & $13.1 \pm 2.7$ & $14.5 \pm 2.5$ & $14.7 \pm 2.8$ & $\begin{array}{l}0.075,0.077, \\
0.082\end{array}$ \\
\hline 23-gauge & $14 \pm 3$ & $12 \pm 5.6$ & $13.9 \pm 3.1$ & $14.9 \pm 2.4$ & $\begin{array}{l}0.035,0.048, \\
0.109\end{array}$ \\
\hline P value $^{\mathrm{b}}$ & 0.910 & 0.390 & 0.459 & 0.757 & \\
\hline
\end{tabular}

Notes: ${ }^{a}$ The three $p$ values calculated by Student's $t$-test are for comparisons of: mean IOP at baseline to that at first postoperative day, mean IOP at the first postoperative day to that at I week postoperatively, and mean IOP at I week postoperatively to that at the final visit, respectively. ${ }^{\mathrm{b}} \mathrm{P}$ value calculated by Student's $t$-test for comparing the mean IOP values in the 27 -gauge to those in the 23 -gauge groups at different time points.

better than that at baseline in each of the groups but there was no significant difference between the two groups at any time point (Table 3). In the 27-gauge group, BCVA improved from a mean of 20/90 at baseline to a mean of $20 / 40$ at 1 month $(\mathrm{p}=0.005)$ and in the 23-gauge group from a mean of $20 / 95$ to $20 / 50(p=0.001)$.

Within 12 months after they received vitrectomy, out of 4 phakic eyes in the 27-gauge group and 7 phakic eyes in the 23 -gauge group at baseline, two eyes $(50 \%)$ and 6 eyes (86\%) developed cataract, respectively $(\mathrm{p}=0.491)$. They all received elective phacoemulsification surgery at a later date. Also, compared to $0 \%$ of the eyes in the 27 -gauge group, 2 eyes from the 23 -gauge group (5\%) had IOP $>25$ on two consecutive visits with 1 eye showing characteristic glaucomatous optic nerve and visual field defects $(\mathrm{p}=0.549)$. In both eyes, IOP was medically controlled up to the final visit.

\section{Discussion}

The introduction of 27-gauge instrument systems represents a new step in the development of MIVS. We compare this novel system with the more readily available 23-gauge

Table 3 Best-Corrected Visual Acuity (BCVA) in LogMAR Units at Different Time Points in Patients Who Had 27-Gauge and 23Gauge Vitrectomy

\begin{tabular}{|c|c|c|c|c|}
\hline & Baseline & $\begin{array}{l}\text { I-Month } \\
\text { Visit }\end{array}$ & Final Visit & p value ${ }^{a}$ \\
\hline 27-gauge & $0.65 \pm 0.47$ & $0.33 \pm 0.21$ & $0.37 \pm 0.22$ & $0.005,0.465$ \\
\hline 23-gauge & $0.68 \pm 0.37$ & $0.39 \pm 0.27$ & $0.42 \pm 0.25$ & $0.00 I, 0.62 I$ \\
\hline$P$ value $^{b}$ & 0.824 & 0.346 & 0.509 & \\
\hline
\end{tabular}

Notes: ${ }^{a}$ The two $p$ values calculated by Student's $t$-test are for comparisons of mean BCVA at baseline to that at I month postoperatively and mean BCVA at I month postoperatively to that at final visit, respectively. ${ }^{b} \mathrm{P}$ values calculated by Student's $t$-test comparing the mean BCVA values in the 27-gauge to those in the 23-gauge groups at different time points. system in terms of the tendency for wound leak or hypotony and the clinical outcomes and complications. To improve statistical reliability, after we retrospectively examined all our 27-gauge cases with several indications, we matched them and compared them to twice as many consecutive patients who had 23-gauge MIVS for the same indications in the same time period and were followed up for at least a year. Furthermore, to limit variability between individual surgeries, all cases were performed under the same surgical settings and by one retina surgeon.

The wound sizes of 20-gauge, 23-gauge, and 25-gauge sclerotomies are approximately $0.9 \mathrm{~mm}, 0.7 \mathrm{~mm}$, and $0.5 \mathrm{~mm}$, respectively. ${ }^{8}$ With 27 -gauge micro-incisions being 20\% smaller than 25-gauge incisions, they are expected to seal better and earlier. To test this property clinically, we examined both the rate of early postoperative hypotony and how frequently sclerotomy stitching was needed at the end of surgery. We found that a statistically significant $18 \%$ of eyes in the 23 -gauge eyes developed hypotony early after surgery, albeit selflimiting, whereas none of our 22 eyes who had 27-gauge vitrectomy did. We also reported that for a statistically significant $25 \%$ of the 23 -gauge eyes compared to none of the eyes in the 27-gauge group, sclerotomy closure by sutures was necessary to finish the surgery. When IOP values directly before and directly after surgery were compared, we noticed a transient but significant drop in the 23-gauge group but not in the 27-gauge group. This is consistent with the fact that more patients developed postoperative hypotony in the 23-gauge group and is in keeping with recent comparative 27 -gauge vitrectomy studies. ${ }^{2}$

Although it has been suggested that angled entry with 27gauge trocars may be preferred over straight entry to avoid transient postoperative hypotonic shifts, ${ }^{9}$ we used a simple oblique 1-step technique for trocar entry in our 27-gauge cases and found no clinical evidence of sclerotomy leak. Sclerotomy-related findings in our 23-gauge cohort are similar to those in other 23-gauge MIVS studies. For example, in a recent 23 -gauge vitrectomy report, $29 \%$ of cases required at least one sclerotomy suture and $14 \%$ of cases had hypotony on the first postoperative day. ${ }^{10}$

Our experience regarding the 27-gauge group is consistent with the notion that 27-gauge micro-sclerotomies have excellent self-sealing properties. In a cohort of 16 patients from Italy who had 27-gauge MIVS for indications similar to those in our patients, none of the patients developed hypotony or needed sclerotomy stitching. ${ }^{11}$ In other 27-gauge cohort studies, however, rates of 
postoperative hypotony between $2 \%$ and $5 \%$ were reported. In a recent cohort with ERM cases, 2\% developed hypotony the next day after surgery although none of the patients needed any sclerotomy stitching. ${ }^{12}$ Similarly, in another report of 95 cases of 27-gauge vitrectomies, stitching was needed in $1 \%$ and postoperative hypotony was documented in $5 \%$ of patients. ${ }^{13}$ It's interesting to note that in that report, all the 5 eyes which had transient hypotony were fluid-filled at the end of surgery. In comparison, all of our cases underwent fluid-air exchange at the conclusion of surgery. Additionally, 3 of those eyes had unique indications, mainly endophthalmitis and silicone oil removal, which were absent in our cohort. In another study of over 400 cases, the authors reported 14 eyes $(3.4 \%)$ who had transient hypotony although no patients needed any stitching at the end of surgery or postoperatively. Unlike our group, however, all of those patients had rhegmatogenous retinal detachment, which represents different pathological, technical, and prognostic characteristics with potentially different hypotony rates and complication profile, especially that the authors reported a similar percentage of hypotony in their cohort even prior to surgery. ${ }^{14}$ The higher rate of hypotony observed in those studies may also be possibly explained by their larger number of cases which carries enough power to detect such a relatively uncommon complication.

It has been suggested that longer vitrectomy times are expected with 27-gauge platforms due to the smaller radius of the cutter's lumen and limited fluid flow rates. $^{12,15-17}$ However, several clinical studies have demonstrated similar operation times in 27-gauge vitrectomy compared to its 23- and 25-gauge counterparts. $3,18,19$ In our study, 23-gauge surgeries actually lasted slightly longer at a mean of 39 mins compared to 27-gauge surgeries at a mean of 35 mins, although the difference was not statistically significant. Moreover, our operative times in 27-gauge cases were consistent with findings from other cohorts with similar indications. ${ }^{11,20}$

Similar to what was reported in 27-gauge and othergauge MIVS before, all of our surgeries were anatomically successful and visual acuity outcomes were excellent with BCVA improving in most eyes. In addition, visual outcomes were not statistically different between the 27 - and 23 -gauge groups and were similar to outcomes reported in 23- and 25-gauge MIVS studies. ${ }^{19,21-23}$

Some studies had a similar design to our study but compared 27-gauge to 25-gauge MIVS. They reported no significant difference between the two platforms in operation time, rate of hypotony, sclerotomy suturing, anatomical outcomes or surgically-induced astigmatism. ${ }^{19,24}$ To the best of our knowledge, our study is the first clinical study to directly compare 27-gauge to the more widely used 23-gauge MIVS patients for clinical outcomes and complications.

Limitations to our study include its retrospective nature with its inherent risks of bias, lack of randomization of patients to the gauge of surgery, small number of our 27gauge cases, and variable follow-up time. Nonetheless, several points of strength in our study include matched indications for surgery, extended post-operative follow-up time to detect any long-term consequences, the exclusion of combined cataract cases and previous vitrectomy cases which may affect operative vitrectomy time and complication rates, and the consistency in the operating surgeon and surgical method and settings for all cases.

\section{Data Sharing Statement}

The datasets generated during and/or analyzed during the current study are available from the corresponding author on reasonable request.

\section{Ethics Approval}

The institutional review board at the University of AlQadisiyah, Diwaniyah, Iraq granted us permission to conduct this study and all participants provided written informed consent (approval \# 03/2019/124). This research complies with the tenants of the Declaration of Helsinki.

\section{Author Contributions}

All authors contributed to conception and design, data analysis, drafting or revising the article, gave final approval of the version to be published, and agree to be accountable for all aspects of the work.

\section{Disclosure}

The authors report no conflicts of interest in this work.

\section{References}

1. Shinoda H, Shinoda K, Satofuka S, et al. Visual recovery after vitrectomy for macular hole using 25-gauge instruments. Acta Ophthalmol. 2008;86(2):151-155.

2. Charles S, Ho AC, Dugel PU, et al. Clinical comparison of 27-gauge and 23-gauge instruments on the outcomes of pars plana vitrectomy surgery for the treatment of vitreoretinal diseases. Curr Opin Ophthalmol. 2020;31(3):185-191. doi:10.1097/ICU.0000000000000659

3. Naruse Z, Shimada H, Mori R. Surgical outcomes of 27-gauge and 25 -gauge vitrectomy day surgery for proliferative diabetic retinopathy. Int Ophthalmol. 2019;39(9):1973-1980. doi:10.1007/s10792-018$1030-\mathrm{z}$ 
4. Saleh OA, Al-Dwairi RA, Mohidat H, et al. International multi-center study of iatrogenic retinal tears in pars plana vitrectomy. Int J Ophthalmol. 2019;12(6):996-1000. doi:10.18240/ijo.2019.06.19

5. Rizzo S, Genovesi-Ebert F, Murri S, et al. 25-gauge, sutureless vitrectomy and standard 20-gauge pars plana vitrectomy in idiopathic epiretinal membrane surgery: a comparative pilot study. Graefes Arch Clin Exp Ophthalmol. 2006;244(4):472-479. doi:10.1007/s00417-005-0173-6

6. ACAR N, KAPRAN Z, UNVER YB, et al. Early postoperative hypotony after 25-gauge sutureless vitrectomy with straight incisions. Retina. 2008;28(4):545-552. doi:10.1097/IAE.0b013e318162b008

7. Thompson JT. Advantages and limitations of small gauge vitrectomy. Surv Ophthalmol. 2011;56(2):162-172.

8. Rizzo S, Patelli F, Chow DR. Vitreo-Retinal Surgery - Essentials in Ophthalmology. Textbook, ISBN 978-3-540-69461-8. Springer Verlag Berlin Heidelberg; 2009.

9. Khan MA, Durrani A, Hsu J, et al. 27-gauge vitrectomy wound integrity: a randomized pilot study comparing angled versus straight entry in fluid-filled vitrectomized eyes. Retina. 2018;38(4):678-683.

10. Küçük E, Yılmaz U, Zor KR, et al. Risk factors for suture requirement and early hypotony in 23-gauge vitrectomy for complex vitreoretinal diseases. Int Ophthalmol. 2017;37(4):989-994. doi:10.1007/ s10792-016-0361-x

11. Rizzo S, Barca F, Caporossi T, et al. Twenty-seven-gauge vitrectomy for various vitreoretinal diseases. Retina. 2015;35(6):1273-1278. doi:10.1097/IAE.0000000000000545

12. Naruse S, Shimada H, Mori R. 27-gauge and 25-gauge vitrectomy day surgery for idiopathic epiretinal membrane. $B M C$ Ophthalmol. 2017;17(1):188. doi:10.1186/s12886-017-0585-1

13. Khan MA, Shahlaee A, Toussaint B, et al. Outcomes of 27 gauge microincision vitrectomy surgery for posterior segment disease. Am J Ophthalmol. 2016;161:36-43. doi:10.1016/j.ajo.2015.09.024

14. Shinkai Y, Oshima Y, Yoneda K, et al. Multicenter survey of sutureless 27-gauge vitrectomy for primary rhegmatogenous retinal detachment: a consecutive series of 410 cases. Graefes Arch Clin Exp Ophthalmol. 2019. doi:10.1007/s00417-019-04448-2
15. Kasi SK, Hariprasad SM, Hsu J. Making the jump to 27-gauge vitrectomy: perspectives. Ophthalmic Surg Lasers Imaging Retina. 2017;48(6):450-456. doi:10.3928/23258160-20170601-02

16. Mori R, Naruse S, Shimada H. Comparative study of 27-gauge and 25-gauge vitrectomy performed as day surgery. Int Ophthalmol. 2018;38(4):1575-1582. doi:10.1007/s10792-017-0625-0

17. Garweg JG, Ouassi D, Pfister IB. Hybrid 23/27 gauge vitrectomy combining the charm of $27 \mathrm{G}$ with the efficacy of $23 \mathrm{G}$. Clin Ophthalmol. 2020;14:299-305. doi:10.2147/OPTH.S233884

18. Pavlidis M. Two-dimensional cutting (TDC) vitrectome: in vitro flow assessment and prospective clinical study evaluating core vitrectomy efficiency versus standard vitrectome. $J$ Ophthalmol. 2016;2016:3849316. doi:10.1155/2016/3849316

19. Mitsui K, Kogo J, Takeda H, et al. Comparative study of 27-gauge vs 25-gauge vitrectomy for epiretinal membrane. Eye (Lond). 2016;30 (4):538-544. doi:10.1038/eye.2015.275

20. Oshima Y, Wakabayashi T, Sato T, et al. A 27-gauge instrument system for transconjunctival sutureless microincision vitrectomy surgery. Ophthalmology. 2010;117(1):93-102. doi:10.1016/j. ophtha.2009.06.043

21. Ibarra MS, Hermel M, Prenner JL, et al. Longer-term outcomes of transconjunctival sutureless 25-gauge vitrectomy. Am J Ophthalmol. 2005;139(5):831-836. doi:10.1016/j.ajo.2004.12.002

22. Oshima Y, Ohji M, Tano Y. Surgical outcomes of 25-gauge transconjunctival vitrectomy combined with cataract surgery for vitreoretinal diseases. Ann Acad Med Singapore. 2006;35:175-180.

23. Fine HF, Iranmanesh R, Iturralde D, et al. Outcomes of 77 consecutive cases of 23-gauge transconjunctival vitrectomy surgery for posterior segment disease. Ophthalmology. 2007;114(6):1197-1200. doi:10.1016/j.ophtha.2007.02.020

24. Jiang $X$, Zhang S, Zhang Z, et al. Comparative study of 27-gauge versus 25-gauge vitrectomy with air tamponade in the treatment of myopic foveoschisis. Ophthalmic Surg Lasers Imaging Retina. 2018;49(10):e135-e142. doi:10.3928/23258160-20181002-16
Clinical Ophthalmology

\section{Publish your work in this journal}

Clinical Ophthalmology is an international, peer-reviewed journal covering all subspecialties within ophthalmology. Key topics include: Optometry; Visual science; Pharmacology and drug therapy in eye diseases; Basic Sciences; Primary and Secondary eye care; Patient Safety and Quality of Care Improvements. This journal is indexed on PubMed
Central and CAS, and is the official journal of The Society of Clinical Ophthalmology (SCO). The manuscript management system is completely online and includes a very quick and fair peer-review system, which is all easy to use. Visit http://www.dovepress.com/ testimonials.php to read real quotes from published authors. 